\title{
Tra docente e apprendente: una biografía linguística
}

Carmela Camodeca

\section{(2) OpenEdition \\ 1 Journals}

Edizione digitale

URL: http://journals.openedition.org/esp/442

DOI: $10.4000 /$ esp.442

ISSN: 2532-0319

Editore

Centre d'Information sur l'Éducation Bilingue et Plurilingue

\section{Edizione cartacea}

Data di pubblicazione: 1 dicembre 2014

Paginazione: 89-99

ISSN: 1127-266X

\section{Notizia bibliografica digitale}

Carmela Camodeca, "Tra docente e apprendente: una biografía linguística », Éducation et sociétés plurilingues [Online], 37 | 2014, Messo online il 01 octobre 2015, consultato il 15 septembre 2020. URL: http://journals.openedition.org/esp/442 


\section{TRA DOGENTE E APPRENDENTE: UNA BIOGRAFIA LINGUISTICA}

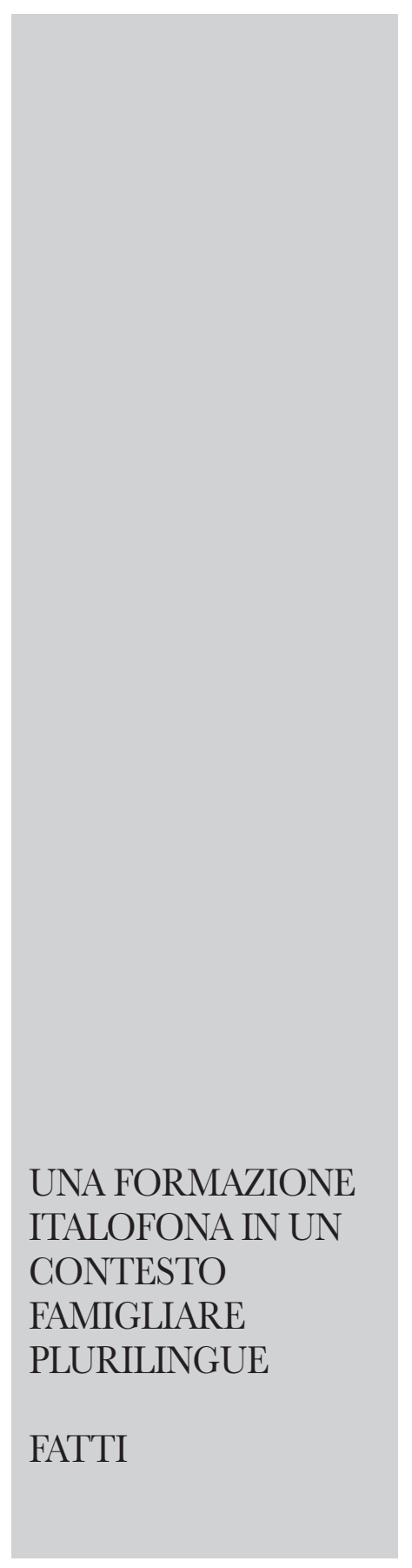

\section{Carmela Gamodeca}

"Il posseder più lingue dona una certa maggior facilità e chiarezza di pensare seco stesso, perché noi [95] pensiamo parlando. Ora nessuna lingua ha forse tante parole e modi da corrispondere ed esprimere tutti gl'infiniti particolari del pensiero. Il posseder più lingue e il potere perciò esprimere in una quello che non si può in un'altra, o almeno cosi acconciamente, o brevemente, o che non ci viene cosi tosto trovato da esprimere in un'altra lingua, ci dà una maggior facilità di spiegarci seco noi e d'intenderci noi medesimi, applicando la parola all'idea che senza questa applicazione rimarrebbe molto confusa nella nostra mente. [...]"

da G. Leopardi, Zibaldone di pensieri, 1819, PP. 53-4 (1)

Q

uesto pensiero di Leopardi, che anticipa di più di un secolo molte delle acquisizioni della moderna linguistica, mi ha suggerito la prospettiva da assumere nel redigere la mia biografia linguistica.

Anch'io, infatti, nel mio modestissimo ambito, ho sperimentato quanto afferma il gigante Leopardi: la possibilità di ricorrere a più codici linguistici, di mettere in atto quei comportamenti che oggi assumono la denominazione di code switching e di code mixing, proprio perché ogni codice singolarmente preso è incompleto e da solo non sufficiente a dar corpo a tutti i significati e le sfumature attraverso i quali può esprimersi l'esperienza individuale e collettiva.

Qualche annotazione per descrivere il retroterra linguistico famigliare e il relativo spazio linguistico. Infanzia e adolescenza trascorse in un paese del cuneese all'imbocco di una valle occitana, dove il dialetto piemontese era il codice di comunicazione dominante e l'italiano un codice alto riservato ai registri formali, veicolare per un numero ristretto di persone: una tipica condizione di diglossia. Un paese che praticava però, per via di un nutrito numero di emigranti, intensi scambi con la Provenza, e dunque il possesso da parte della popolazione di una competenza perlome- 
Tra docente e apprendente: una biografía linguística

C. CAmodeca

... RICORDI E RIFLESSIONI

TRE LINGUE EUROPEE. UNA BASE COMUNE E TRE DIFFERENTI MODALITÀ DI ACQUISIZIONE no passiva del francese.

Padre (distante per motivi di lavoro), originario di una comunità alloglotta albanese dell'Alto Ionio, di madrelingua arbëreshë, italofono per studi e cultura famigliare e lusofono causa un ventennio trascorso in Brasile tra le due guerre. Madre piemontese di nascita e di madrelingua (ma occitana per parte di madre), italofona per formazione scolastica e per la sua professione di insegnante elementare; con un'ottima conoscenza - e soprattutto un grande amore - per la lingua francese, imparata in contesto scolastico e costantemente coltivata; con conoscenza dello spagnolo, appreso da autodidatta, e di qualche rudimento di tedesco, per tristi vicissitudini di prigionia.

Lingua veicolare in famiglia, l'italiano, di registro medio alto, ma con 'incastri' di dialetto piemontese e delle altre lingue citate: in piemontese proverbi, battute, filastrocche, ecc.., in altre lingue, tra cui primeggiava il francese, frasi ricorrenti.

Questa nostra italofonia in un universo dialettofono e l'appartenenza a una famiglia percepita dal contesto locale come distante dalla norma ci ha in parte rese, noi due figlie, diverse: tutti, grandi e piccoli, parlavano tra di loro il piemontese ma si rivolgevano a noi esclusivamente in italiano. Come se obbedissimo ad un'implicita censura, questo atteggiamento ci ha impedito lo sviluppo della competenza attiva del dialetto piemontese.

Ricordo, delle altre lingue, parole sparse impiegate nella narrazione di vicende, nella denominazione di oggetti particolari, nel commento di vecchie foto; lettere in portoghese; le pagine ingiallite di una grammatica arbëreshë per ginnasiali; l'albero genealogico della famiglia redatto in greco e albanese. E un lessico famigliare con forte presenza del francese, che continuavamo ad arricchire durante i nostri regolari soggiorni in Francia.

Ho acquisito tre lingue europee, il francese, l'inglese e il tedesco, in fasce d'età e contesti differenti, e con metodi, tecniche e materiali dissimili. In comune: un lessico a base latina, alcune strutture riconoscibili, strategie cognitive già sperimentate, poi abbandonate o rimodulate.

Sono insegnante (al presente, di italiano L2) e non posso esporre la mia biografia linguistica se non dall'ottica per me dominante, la riflessione sull'acquisizione delle lingue seconde; è questa che mi fornisce i parametri attraverso i quali organizzare dati autobiografici spesso frammentari e disomogenei. Perciò, ho scelto di porre in evidenza un solo aspetto di ognuna delle tre lingue in 
Tra docente e apprendente: una biografía linguística

C. Camodeca

\section{IL CASO DEL FRANCESE: UN'ACQUISIZIONE PRECOCE}

\section{FATTI}

RICORDI

E RIFLESSIONI questione, quello che maggiormente lo identifica:

- nel caso del francese, l'acquisizione precoce

- nel caso del tedesco, l'acquisizione in contesto linguistico-culturale germanofono

- nel caso dell'inglese, l'apprendimento in contesto formale e la contemporanea maturazione di un'esperienza glottomatetica.

Sono stata esposta alla lingua e alla cultura francese dal 1949 al 1958, dai due fino agli undici anni, per tre mesi consecutivi durante il periodo estivo, in una città del nord est della Francia, dove risiedevamo a causa dell'impiego paterno presso il locale Consolato italiano.

A partire dai sette anni, mi è stato anche impartito un insegnamento formale della lingua (lettura e scrittura): tre ore di lezione ogni mattina. Nel frattempo, la lettura autonoma dei primi i libri per l'infanzia; alcune stabili amicizie con coetanei del luogo.

Durante il resto dell'anno, che trascorrevo in Italia, continuavo a praticare la lettura (ero, per esempio, abbonata a Le journal de Mikey, il corrispettivo del Topolino italiano) e la scrittura (regolare corrispondenza con i miei amichetti francesi).

Mi sono resa conto solo in seguito di aver vissuto in quegli anni esperienze culturali estremamente significative sul piano cognitivo-affettivo. Riporto un elenco, volutamente disordinato, delle impressioni che ho conservato:

Le lunghe e complicate operazioni di frontiera alla dogana Bardonecchia-Modane negli anni dell'immediato dopoguerra; la celebrazione del 14 Juillet, un rito immancabile, con gran raduno di gente per i fuochi d'artificio; le scritte sui muri degli anni della guerra d'Algeria; i numerosi vicini americani, con i loro macchinoni, sempre sorridenti ma impenetrabili; i parchi pubblici con il culto delle aiuole fiorite e i bambini sfreccianti sulle trottinettes; il teatrino con Guignol; i grandi magazzini; le case con i tetti spioventi e i nidi di cicogna sui camini, le scale a chiocciola, le elegantissime palazzine liberty in rue Emile Gallé; le vetrate delle cattedrali gotiche; i cibi e le bevande con i loro odori e sapori: le brioches, la choucroute, le crêpes, l'odore aleggiante della birra; i negozietti di bric-à-brac, con i vasi art nouveau e déco della Verrerie Daum e i soldatini di piombo dell'armata di Napoleone schierati nelle vetrinette Luigi XV; i grandi canali navigabili e il lentissimo passaggio delle chiatte tirate dai cavalli; la maestra di francese, anziana signorina perennemente sorridente, serafica, prodiga di gratificazioni "Très bien! Très, très bien!; le illustrazioni dei miei libri di lettura; i film di 
Tra docente e apprendente: una biografía linguística

C. CAmodeca
IL CASO DEL
TEDESCO:
L'ACQUISIZIONE IN
CONTESTO
LINGUISTICO-
CULTURALE
GERMANOFONO

FATTI

RICORDI

E RIFLESSIONI

\section{Walt Disney: Peter Pan, Cendrillon, La belle et le clochard.}

Dunque: la mia competenza passiva della lingua si è formata in tenerissima età mentre sono stata alfabetizzata (lettura e scrittura) quasi contemporaneamente nelle due lingue.

Per l'insegnamento del francese la nostra 'precettrice' applicava gli stessi metodi e materiali utilizzati nell'insegnamento a bambini di madrelingua; e fortunatamente venivano a mancare gli aspetti particolarmente ansiogeni del contesto scolastico (prove in classe, voti, competizione con gli altri scolari...); questo ha indubbiamente inciso sulla dimensione affettiva dell'apprendimento e sul mio successivo rapporto con la lingua francese.

Tuttavia, la progressione in ognuna delle due lingue è avvenuta in contesti separati e differenziati non solo per caratteristiche linguistico-culturali ma anche spazio-temporali (l'una in una città, l'altra in un paese; l'una concentrata in tre mesi, l'altra per il resto dell'anno) e relazionali (gli amici francesi, gli amici italiani, senza contatto reciproco). Lo erano anche nella mia rappresentazione mentale.

Nel contempo ho inconsapevolmente maturato una competenza biculturale e muovermi tra due culture ha costituito la naturale dimensione della mia esistenza; ricordo però che cercavo di cancellare la mia dimensione francese al ritorno in Italia, perché temevo il rifiuto dei miei coetanei.

Subito dopo il conseguimento della laurea (Roma, 1970), ho deciso di prendermi un cosiddetto 'anno sabbatico' e mi sono trasferita nell'allora Repubblica Federale Tedesca. Due mesi prima della partenza, ho iniziato lo studio del tedesco da autodidatta.

A Francoforte sul Meno, ho vissuto presso due famiglie per un periodo di quattro mesi, in totale immersione linguistico-culturale. Impossibilitata per motivi di lavoro a frequentare un regolare corso di lingua, ho continuato per conto mio, con una certa sistematicità, lo studio del tedesco. Durante l'estate, ho finalmente potuto frequentare un corso di ferie per stranieri, presso la Fohannes Wolfgang Goethe Universität.

Ancora in Italia, nel mio primo approccio alla lingua tedesca, abituata ad un insegnamento linguistico di tipo traduttivo-grammaticale, mi sono affidata ad una grammatica che mi fornisse una completa descrizione della lingua. L'idea allora diffusa secondo la quale il tedesco, come il latino, è una lingua particolarmente 'logica' e quella, allora altrettanto comune, secondo la quale 'se si sa la grammatica si sa anche parlare' ha rafforzato questa mia scelta. 
Tra docente e apprendente: una biografía linguística

C. Camodeca
Arrivata in loco è stata la morfologia flessiva l'area più problematica; in misura minore la sintassi, data la rigidità della struttura della frase tedesca. Così, mentre la conoscenza teorica non mi ha agevolata nell'acquisizione della morfologia flessiva, mi ha invece permesso di acquisire precocemente la capacità di costruire frasi sintatticamente corrette. Del lessico, invece, ricordo che ero in grado di riconoscere nel parlato i vocaboli a me noti e che ciò mi creava sicurezza. Sotto il profilo fonetico, non ho avuto difficoltà nell'acquisizione dei fonemi non appartenenti al sistema fonologico dell'italiano; questo grazie anche alla conoscenza del francese ed alla mia provenienza da un'area regionale in cui, per esempio, i fonemi vocalici /ü/ e /ö/ sono presenti nel dialetto; ricordo di aver anche acquisito abbastanza precocemente la consapevolezza della distinzione tra vocali brevi e lunghe.

La mia competenza linguistica è proceduta di pari passo con quella comunicativa e con quella extralinguistica in tutti i suoi aspetti; ricordo per esempio di aver prontamente assimilato la tipica gestualità dei nativi. Tuttavia non riuscivo a superare l'ansia dell'errore: l'errore, secondo quello che era allora il punto di vista corrente, era indice di incultura e poteva generare riprovazione sociale, quasi come se, 'trattando male la lingua', si facesse altrettanto con le persone. Avrebbe invece dovuto rassicurarmi il fatto che il mio modo di esprimermi era considerato formalmente molto corretto; forse per questo motivo, mi sembra di ricordare, nessuno si è mai rivolto a me utilizzando una qualsiasi forma di foreigner talk.

Sul piano culturale, provavo una forte curiosità per la cultura materiale: il cibo, le modalità abitative, l'uso del tempo libero (tutti, appena spuntava un raggio, a prendere il sole nei parchi!), le ricorrenze (gli addobbi natalizi e pasquali, la festa del Maikäfer, il maggiolino) ed ho scelto di far miei i tratti che sentivo affini. Questo processo di integrazione è stato a mio avviso agevolato da due fattori: da un lato la mia ancora relativa plasticità 'culturale', derivante dalla mia giovane età, ancora in fase di ricerca di un proprio stile di vita; dall'altro, la condivisione di valori e mode comuni all'allora mondo giovanile dell'Europa occidentale, plasmato dalle trasformazioni socio-culturali di fine anni sessanta.

Ma la scoperta più degna di nota è stata quella dell'immigrazione - italiana, ma anche spagnola, portoghese, turca -, il realizzare che si stava formando una società multietnica. 
Tra docente e apprendente: una biografía linguística

C. Camodeca

\section{FATTI}

\section{RICORDI E RIFLESSIONI}

Nell'ottobre dello stesso anno (1971), ho avuto un incarico di insegnamento in una cittadina nei pressi di Francoforte, presso una scuola tedesca, in una classe di bambini italiani di recente immigrazione. Classi del genere, denominate Einfuihrungsklassen (classi di inserimento), avevano lo scopo di far 'acclimatare'i bambini stranieri prima che fossero inseriti nelle corrispettive classi tedesche e prevedevano un insegnamento nelle propria madrelingua con l'aggiunta di ore di tedesco, non sempre effettuate.

Durante questo mio secondo soggiorno, ho stabilito la mia residenza in un appartamentino presso una famiglia tedesca e ho regolarmente frequentato, per sei ore settimanali, un corso di lingua per studenti stranieri. Nel frattempo ho approfondito la mia conoscenza della cultura tedesca, con grande interesse per il particolare momento storico che quel paese stava vivendo (erano gli anni della Ostpolitik e del cancellierato di Willy Brandt); inoltre sono venuta più profondamente a contatto con la realtà dell'immigrazione italiana e ne ho seguito le vicende.

Un'appendice a questa mia esperienza è stata la frequenza di un corso di ferie, della durata di un mese, questa volta nell'allora DDR, la Repubblica Democratica Tedesca, presso la Pädagogische Hochschule di Erfurt.

Il corso di lingua tedesca che frequentavo era condotto da un assistente di germanistica e seguito da una ventina di studenti di varie nazionalità; era teso a sviluppare soprattutto le abilità ricettive, di comprensione e produzione di testi scritti; poco considerata la produzione orale. Le lezioni erano condotte in modo frontale; l'approccio adottato era di tipo testuale. Il materiale consisteva in due raccolte di testi, rispettivamente letterari e non letterari, su cui erano impostati esercizi di natura grammaticale e lessicale; raramente veniva usato materiale audio.

Questo periodo ha coinciso col mio ingresso nel mondo del lavoro vero e proprio: un contratto e un rapporto di lavoro stabile, le garanzie sociali, uno stipendio regolare, l'affitto di un appartamentino a proprio nome, il primo conto in banca. I primi passi verso 'l'adultità' (con i suoi apprezzabili vantaggi, quali l'autonomia e l'indipendenza economica) sono dunque per me avvenuti in un contesto socio-culturale non italiano.

Difficile e complesso invece il contesto lavorativo: un'estrema difficoltà a gestire una classe di trenta ragazzini, dagli otto ai tredici anni, senza nessuna preparazione pedagogica specifica da parte mia; ma contemporaneamente il mio tentativo di non fare della mia classe un ghetto, di farla partecipare alle attività della scuola (uso delle aule speciali, recite scolastiche, partecipazione a corsi opzionali come le ore di musica...). In particolare, lo sforzo per far sì che la lingua tedesca le fosse insegnata da un madrelingua (in quegli anni la scuola tedesca soffriva di una grave penuria di insegnanti). Riuscita, in quest'ultimo tentativo, ho io stessa partecipato, in veste di alunna e di 'mediatrice', a queste lezioni: il materiale uti- 
Tra docente e apprendente: una biografía linguística

C. CAmodeca
FATTI lizzato era concepito per madrelingua e venivano impiegati metodi propri della didattica ludica.

Nel contempo, mi adoperavo per intessere relazioni positive con il corpo insegnante della scuola, applicando idonee strategie pragmatico-comunicative. In quell'occasione ho maturato la consapevolezza che, per instaurare una comunicazione interculturale soddisfacente, lo sforzo deve essere reciproco; avevo davanti a me l'esempio di altre insegnanti italiane che avevano un atteggiamento di chiusura nei confronti dei colleghi nativi (per esempio trascorrevano gli intervalli 'barricate' nella loro aula, invece che nella comune aula insegnanti). Forse, i tempi non erano ancora maturi e la condizione di 'immigrata' poteva ancora essere vissuta con disagio.

Ora però mi rendo conto che io stessa, per altri versi, ho dovuto fare $\mathrm{i}$ conti con il 'fantasma mentale' dell'immigrata: soprattutto nel tentativo di esorcizzarlo. Per esempio, nelle strette relazioni instaurate con la famiglia presso la quale risiedevo, un mio obiettivo era quello di dimostrare nei fatti che gli italiani (qui usato come sinonimo di stranieri) erano corretti, educati, precisi, puliti, ecc... come e più dei nativi. Questo non significa però che io vivessi la lingua italiana come lingua di scarso prestigio, anzi ero fiera del suo statuto di 'lingua di cultura'.

La 'competenza migratoria' da me acquisita, ossia la consapevolezza della condizione dellimmigrato e delle grandi trasformazioni culturali e sociali in atto, mi ha fatto vivere con circa trent'anni di anticipo quanto avviene da qualche decennio in Italia.

Infine, l'esperienza di un mese di vita in DDR, molto più importante sotto il profilo socio-culturale che linguistico, è stata troppo intensa perché possa essere 'compressa' in poche righe, perciò tralascio di riferirne.

Nel 1972 ho intrapreso la mia carriera di insegnante di italiano, prima a Torino e in seguito in Valle d'Aosta, dove ho ristabilito un contatto più intenso con la lingua francese e ho acquisito la competenza passiva del patois francoprovenzale, madrelingua e lingua del contesto famigliare di mio marito.

Nel 1991 ho iniziato l'insegnamento presso il locale Liceo Linguistico, dove ho instaurato un'intensa collaborazione con le colleghe di francese, tedesco, inglese e latino. Ho così partecipato a progetti europei, scambi di classe, soggiorni-studi o semplici viaggi di istruzione all'estero. Di queste numerose attività, la più significativa per quanto riguarda la lingua tedesca è stata, nel 1992, la partecipazione al progetto TEX, (Teacher Exchange), consistente in uno scambio tra docenti di due paesi della CE. Ho quindi soggiornato per un mese nella cittadina di Siegburg (nei pressi di Bonn) partecipando alle attività di un Gymnasiums cittadino, dove era fra l'altro funzionante un corso bilingue 
Tra docente e apprendente: una biografía linguística

C. Camodeca

... RICORDI E RIFLESSIONI

ILCASO DELL'INGLESE: L'APPRENDIMENTO IN CONTESTO FORMALE E IL RAFFORZAMENTO DELLA COMPETENZA GLOTTOMATETICA.

FATTI

RICORDI E RIFLESSIONI franco-tedesco. La mia partner di scambio ha successivamente partecipato, sempre per un mese, alle attività didattiche della nostra scuola.

Il soggiorno nel Gymnasium Alleestrasse è stato per me un vero 'banco di prova', rispetto alla mia capacità di uso veicolare della lingua. Ho svolto la funzione di osservatrice, a volte partecipante, in particolare per le discipline dell'area linguistico-letteraria. La conoscenza del sistema scolastico tedesco ed i contenuti disciplinari sviluppati con i colleghi della scuola hanno arricchito il mio lessico, soprattutto settoriale.

Mi ha fatto riflettere la creatività linguistica della mia partner di scambio quando si esprimeva in italiano, il suo continuo uso di strategie di conseguimento, il suo continuo ricorso al code mixing. Sono rimasta colpita dal fatto che anche parlanti con un alto livello culturale, addirittura con competenze filologiche, non si preoccupassero della correttezza formale delle proprie produzioni orali in lingua straniera, ma si ponessero come obiettivo primario lo scambio comunicativo, concentrati sull'esecuzione piuttosto che sulla progettazione. Di fronte a questi esempi la mia 'ansia dell'errore' si è notevolmente mitigata: ho cominciato a non 'osservarmi' più, mentre parlavo, a non avvertire più come 'disturbanti' le interferenze interlinguistiche. Stavo entrando nella prospettiva della competenza linguistico-comunicativa, che presto si sarebbe affermata attraverso $i$ ben noti documenti europei.

Infine, i successivi soggiorni linguistici mi hanno permesso di vivere più direttamente un altro momento straordinariamente coinvolgente della storia tedesca, quello della Wiedervereinigung, ossia della riunificazione delle due Germanie.

Ho iniziato lo studio scolastico della lingua inglese all'età di quattordici anni, per tre ore settimanali, per i due anni del ginnasio (1961-63). Secondo i programmi di allora, il primo anno era dedicato unicamente alla lingua, nel secondo veniva introdotto lo studio della letteratura.

Di questo periodo ricordo soprattutto un fatto emblematico: la nostra insegnante era di madrelingua e di nazionalità inglese ma conduceva le lezioni rigorosamente in italiano, provando un certo disagio se le succedeva di esprimersi liberamente in inglese al di là dei momenti canonici (lettura delle frasi del libro di testo).

Il metodo applicato era quello traduttivo-grammaticale: frasi, frasi e poi ancora frasi; qualche brevissimo testo alla fine di ogni 
Tra docente e apprendente: una biografía linguística

C. CAmodeca

\section{FATTI}

... RICORDI

E RIFLESSIONI capitolo. Né il libro di testo né tanto meno l'insegnante trattavano argomenti di civiltà, il massimo a riguardo era la descrizione del breakfast.

Tuttavia, a differenza dei libri di greco e di latino, il nostro manuale conteneva delle vignette, figurine stilizzate, a illustrazione del materiale grammaticale e lessicale: cosi, lo percepivamo come meno 'serio' rispetto agli altri, poiché non ne afferravamo la valenza didattica.

Per lo studio della letteratura ci venivano fatti sottolineare ed imparare a memoria gli eventi più rilevanti della biografia dei vari scrittori, da Shakespeare a Wilde, e il titolo e contenuto delle loro opere principali; in più, la lettura di qualche testo, ben al di sopra del nostro livello di competenza.

Dietro la facciata di un discreto andamento scolastico, non ho sviluppato alcuna abilità, salvo una basilare competenza ortografica e lessicale.

Dal 1963 al 1993, i miei rapporti con la lingua e cultura inglese hanno più o meno seguito le vicende della progressiva affermazione in Europa della cultura anglo-americana (musica, cinema, letteratura, ecc).

Nel 1993, sorretta da una forte motivazione strumentale, ho deciso di riprendere lo studio della lingua in un contesto formale guidato; mi sono perciò iscritta ad una scuola di lingua e ho frequentato un corso di quattro ore settimanali per due anni consecutivi. Al termine ho sostenuto l'esame del primo livello certificatorio FCE (First Certificate in English).

Le classi del corso di inglese erano composte da sette-otto allievi, tutti monolingui, ma differenti per età, preparazione scolastica e culturale; il gruppo maggiore era rappresentato da studenti di scuola secondaria. Noi apprendenti eravamo disposti a gruppetti di due-tre, in modo da creare tre o quattro postazioni; ci veniva consigliato di non formare dei gruppetti fissi, ma di fatto ciò avveniva. Il compito dell'insegnante consisteva nel seguire i gruppi nelle varie attività previste dal libro di testo e nell'intervenire con brevi spiegazioni, soprattutto di carattere grammaticale, indirizzate alla classe intera. Durante il lavoro dei gruppi veniva trasmessa della musica di sottofondo, atta a favorire l'instaurarsi di un clima rilassato, favorevole all'apprendimento e ad impedire il disturbo reciproco.

I libri utilizzati erano organizzati in unità didattiche, che venivano integrate con altro materiale, soprattutto audio e video. Le unità 
Tra docente e apprendente: una biografía linguística

C. Camodeca

\section{FATTI}

RICORDI

E RIFLESSIONI miravano allo sviluppo di tutte e quattro le abilità fondamentali, con prevalenza per quella di produzione orale. Questa modalità di gestione della classe e metodologia di insegnamento, che ora è prassi corrente, rappresentava per me una novità. E così ben presto la motivazione strumentale da cui ero partita si è trasformata in una motivazione basata sul piacere.

Potrei affermare che il mio processo di apprendimento non si è svolto in modo progressivo e lineare, ma per 'salti': per esempio, per tutto il primo anno ho avuto l'impressione di non riuscire ad 'entrare' realmente nella lingua. Non mi riusciva di 'entrare' nella fonetica e nella prosodia della lingua inglese - il tedesco stava sempre lì, minaccioso! - e questo ostacolava la comprensione orale; poi, ad un certo punto, ho cominciato ad avvertire nella lingua regolarità ed armonia e il 'blocco' si è allentato.

Un problema di natura socio-affettiva che ho dovuto affrontare è stato quello di dover riconvertire il mio ruolo istituzionale: non solamente ritrovarmi io, insegnante, nelle vesti di alunna, ma dover spesso lavorare con studenti veri, spesso di mia conoscenza o allievi della mia scuola; il problema era naturalmente reciproco. A volte ho dovuto essere particolarmente vigile e controllare la tendenza a rivestire istintivamente il mio ruolo abituale, soprattutto quando venivano condotte riflessioni di carattere metalinguistico o dovevamo fare ricorso alla nostra 'conoscenza del mondo'. Il fatto però di ritrovarsi in territorio 'neutro' ed in una situazione paritaria, con un obiettivo comune, è stata un'esperienza positiva per entrambi.

Negli stessi anni, ho partecipato a tre soggiorni studio ad Exeter, in Inghilterra, come insegnante accompagnatrice di classi di studenti. In tali occasioni ho frequentato dei corsi intensivi nella scuola stessa, risiedendo presso famiglie del luogo.

Durante i soggiorni di studio, ho trasferito l'apprendimento in un contesto anglofono e lì è avvenuto il vero primo salto qualitativo. Sul piano metodologico, la scuola non era tanto diversa dal mio corso di lingua, ma lo era su quello della competenza professionale degli insegnati e delle attrezzature didattiche (biblioteca, videoteca, laboratorio linguistico, aula computer...); inoltre le classi erano plurilingui. Tra le componenti della motivazione basata sul piacere, che continuava a sostenermi, quella ludica ha assunto una netta preminenza, proprio per il tipo di tecniche impiegate nel corso delle lezioni.

Le famiglie che mi hanno ospitata durante i soggiorni erano costi- 
Tra docente e apprendente: una biografía linguística

C. CAmodeca tuite da donne di età più o meno pari alla mia: questa coincidenza mi ha offerto maggior possibilità di scambi comunicativi. Tuttavia, la paura di non capire i miei interlocutori durante le interazioni comunicative era per me il fattore più ansiogeno: per paura di tediare il/la partner con troppe negoziazioni di significato, ho spesso preferito, pur controvoglia, sacrificare la comprensione per salvare la coesione e non lasciar cadere la conversazione. Mi è stato però di aiuto il mio reale e non simulato interesse per la cultura locale, che i miei interlocutori avvertivano.

\section{PER CONGLUDERE}

Attraverso un percorso guidato della memoria, ho cercato di mettere in luce i principali processi cognitivi e socio-affettivi che hanno segnato il mio percorso di acquisizione del francese, del tedesco e dell'inglese. Un percorso di lunga durata, ma che ora sembra remoto. Perché, nel frattempo, oltre alla mobilità che contrassegna la nostra epoca, l'uso delle nuove tecnologie e quindi la possibilità di un contatto linguistico ininterrotto con un mondo globalizzato e multilingue sembrano aver provocato una cesura con i decenni precedenti. Una cesura che ha spazzato via ansie, resistenze, preconcetti, diffidenze, prima tra tutte quella nei confronti del contatto interlinguistico.

Ora, l'accesso alle lingue sembra così immediato e naturale: facilità di spostamenti, materiali in abbondanza e di qualsiasi tipo, contenuti di civiltà declinati in tutti i modi, possibilità di instaurare la comunicazione interlinguistica ovunque e in tempo reale. Eppure, mi chiedo se il senso di sicurezza e di fiducia nella mia competenza comunicativa che provo allo stato attuale sarebbero tali se, quando avevo meno di due anni, non avessi sgranato gli occhi davanti alla locomotiva a vapore della linea ferroviaria Modane-Dijon e non fossi rimasta colpita dalle voci delle guardie di frontiera: Ici douane française! Avez-vous quelque chose à déclarer?

NOTA

(1) LEOPARDI G., Tutte le opere, vol. II, Firenze, Sansoni, 1983. 